\title{
Structural determination of the Dicer-2・R2D2 complex
}

\author{
Helen Donelick, Peter Shen and Brenda Bass
}

University of Utah, Salt Lake City, Utah, United States

In Drosophila melanogaster, Dicer-2 is essential for multiple RNA interference functions, including small interfering RNA (siRNA) biogenesis. These siRNAs are 21-23 nucleotides in length ${ }^{1}$, and one strand is loaded onto Argonaute- 2 to form the active RNA-induced silencing complex (RISC). Loading the guide strand onto Argonaute- 2 requires the formation of the RISC loading complex, which includes Dicer-2, siRNA, and R2D2, a dsRNA binding protein. R2D2 appears to interact with the Dicer-2 helicase domain ${ }^{2}$. The Dicer-2・R2D2 complex binds to siRNA with a high affinity in an ATP independent manner (Figure 1). Without R2D2, siRNAs are generated but cannot be passed on to Argonaute-2, resulting in nonfunctional RNAi. Therefore, R2D2 is essential for RNA interference ${ }^{3,4}$. Although the RISC loading complex is vital for RNA interference, little is known about how this complex passes the guide strand, and no structural information exists on how the complex is formed. It has been suggested that the helicase domain of many Dicer proteins is the domain where accessory proteins can bind and modulate Dicer's function $^{5}$. A high-resolution structure of this complex will provide details into how R2D2 modulates the function of Dicer-2, which can then be validated with biochemical techniques. To acquire a structure, we built on cryo-EM work performed by my co-mentors. We performed single-particle analysis on a dataset collected on the University of Utah Titan Krios. The complex consisted of Dicer-2•R2D2, siRNA, and ATP. Our preliminary analysis of this structure shows an extra density on the helicase domain, not accounted for by an apo-Dicer- 2 model ${ }^{6}$. It appears this extra density on the helicase domain is R2D2, but the current resolution is only at $6.0 \AA$, which makes accurate fitting of this additional density challenging. Thus, exactly how R2D2 regulates Dicer-2 remains unknown. We are continuing to analyze our dataset and utilize Topaz ${ }^{7,8}$ to help find underrepresented views of the complex. With additional data collection and analysis, we feel confident we can obtain a high-resolution structure of this complex and find key domain interactions that we can mutate and test biochemically. This will help provide us with details into mechanistically how R2D2 modulates the function of the Dicer-2 helicase domain, which we can potentially correlate the how accessory proteins modulate human Dicer. Furthermore, this reconstruction could not only help us better understand how siRNAs are passed to Argonaute-2 but could provide preliminary details into is multiple accessory co-factors can bind the Dicer-2 helicase domain simultaneously, which is currently unknown. Based on the location of the proposed R2D2 density, R2D2 may sit on end the helicase domain so when Dicer-2 cleaves the dsRNA into a siRNA, and R2D2 can bind, the siRNA is better positioned to move the siRNA away from the Dicer-2 cleavage site and onto Argonaute-2. 


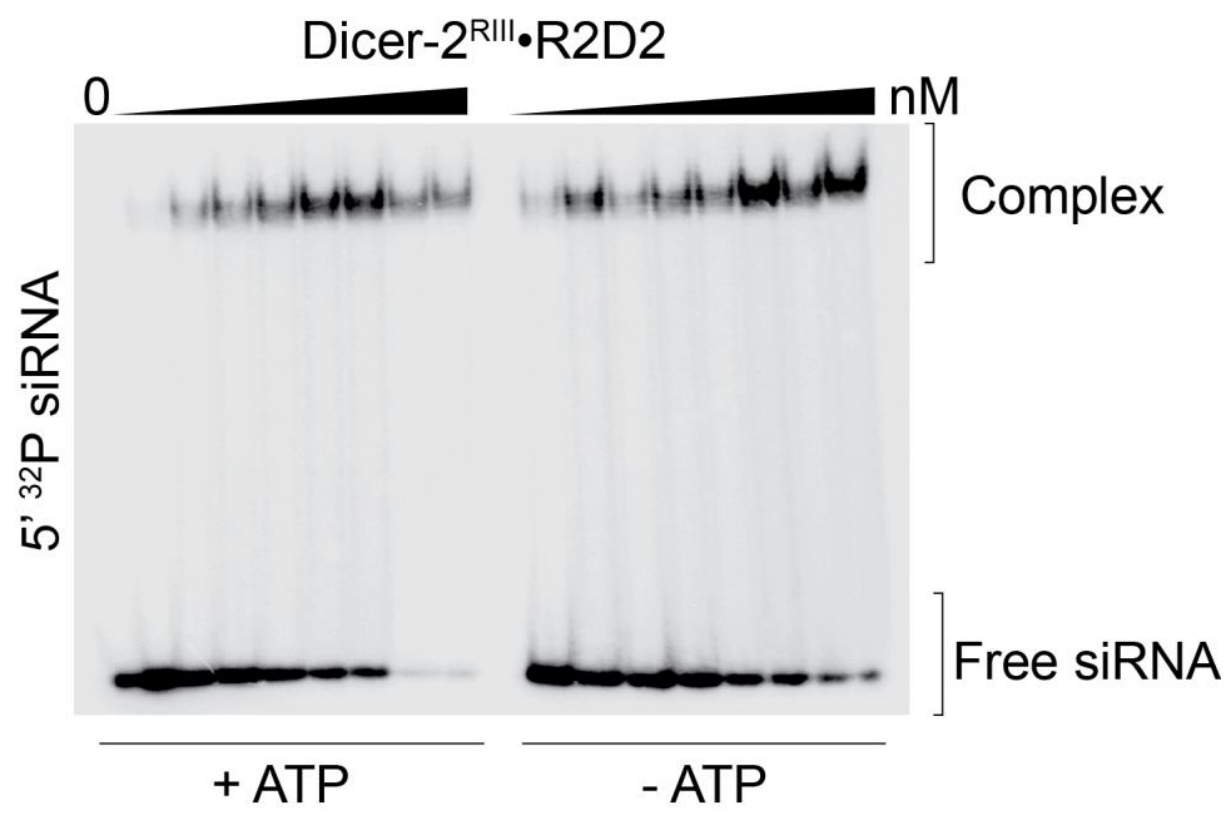

Figure 1. Representative native polyacrylamide gel for an electrophoretic mobility shift assay with 5'32P labeled siRNA incubated with increasing concentrations of Dicer-2•R2D2 +/- 5mM ATP. RIII indicates mutations in the RIII domain to prevent cleavage. Kd of complex binding siRNA is $4 \mathrm{nM}$.
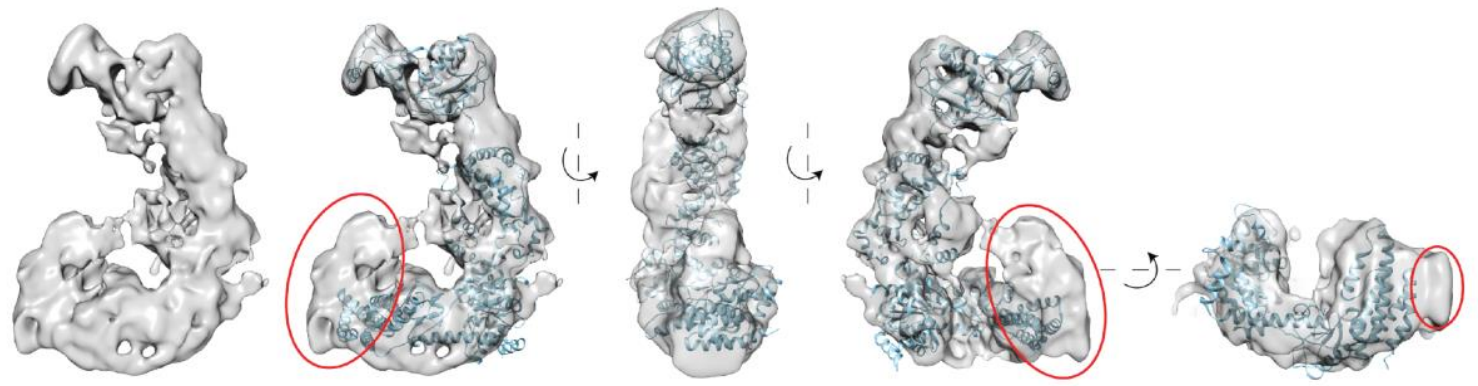

Figure 2. $6.0 \AA 3 \mathrm{D}$ reconstruction of the Dicer-2 $-\mathrm{R} 2 \mathrm{D} 2$ complex. Reconstruction fit with apo-Dicer-2 (6BUA, Sinha et al. 2018). The red circles indicate the extra density not taken into account by apo-Dicer2; this extra density is likely R2D2, but may include part of the siRNA as well, but with the current resolution at $6.0 \AA$ it is difficult to accurately model this density.

\section{References}

1. Elbashir, S. M., Lendeckel, W. \& Tuschl, T. RNA interference is mediated by 21- and 22-nucleotide RNAs. Genes Dev. 15, 188-200 (2001).

2. Nishida, K. M. et al. Roles of R2D2, a Cytoplasmic D2 Body Component, in the Endogenous siRNA Pathway in Drosophila. Mol. Cell 49, 680-691 (2013).

3. Liu, Q. et al. R2D2, a bridge between the initiation and effector steps of the Drosophila RNAi pathway. Science (80-. ). 301, 1921-1925 (2003).

4. Liu, X., Jiang, F., Kalidas, S., Smith, D. \& Liu, Q. Dicer-2 and R2D2 coordinately bind siRNA to promote assembly of the siRISC complexes. Rna 12, 1514-1520 (2006).

5. Hansen, S. R., Aderounmu, A. M., Donelick, H. M. \& Bass, B. L. Dicer's Helicase Domain: A Meeting Place for Regulatory Proteins. Cold Spring Harb. Symp. Quant. Biol. 84, 039750 (2020). 
6. Sinha, N. K., Iwasa, J., Shen, P. S. \& Bass, B. L. Dicer uses distinct modules for recognizing dsRNA termini. Science (80-. ). 359, 329-334 (2018).

7. Bepler, T. et al. Positive-unlabeled convolutional neural networks for particle picking in cryoelectron micrographs. Nat. Methods (2019) doi:10.1038/s41592-019-0575-8.

8. Bepler, T., Kelley, K., Noble, A. J. \& Berger, B. Topaz-Denoise: general deep denoising models for cryoEM and cryoET. Nat. Commun. (2020) doi:10.1038/s41467-020-18952-1. 